\title{
Differentiable Families of Planar Bimodal Linear Control Systems
}

\author{
Josep Ferrer, M. Dolors Magret, and Marta Peña \\ Departament de Matemàtica Aplicada I, ETSEIB, Universitat Politècnica de Catalunya, Diagonal 647, 08028 Barcelona, Spain \\ Correspondence should be addressed to Marta Peña; marta.penya@upc.edu
}

Received 18 October 2013; Revised 19 December 2013; Accepted 19 December 2013; Published 22 January 2014

Academic Editor: Hamid Reza Karimi

Copyright (c) 2014 Josep Ferrer et al. This is an open access article distributed under the Creative Commons Attribution License, which permits unrestricted use, distribution, and reproduction in any medium, provided the original work is properly cited.

\begin{abstract}
We consider bimodal linear control systems consisting of two subsystems acting on each side of a given hyperplane, assuming continuity along it. For a differentiable family of planar bimodal linear control systems, we obtain its stratification diagram and, if controllability holds for each value of the parameters, we construct a differentiable family of feedbacks which stabilizes both subsystems for each value of the parameters.
\end{abstract}

\section{Introduction}

Piecewise linear control systems (in particular, the bimodal ones: see, for example, [1-3]) have attracted the interest of the researchers in recent years, as a special class of switched systems (see, e.g., [4-6]), by their wide range of applications, as well as by the possible theoretical approaches, even in the planar case (see, e.g., [7])

Bimodal linear control systems (BLCS) consist of two subsystems acting on each side of a given hyperplane, assuming continuity along the separating hyperplane. These systems present a complex dynamical behaviour, even for low dimensions, as has been shown in several works. For example, in [8], it is proved that a planar bimodal linear system is stable if each subsystem is stable, but this does not hold for a bimodal linear system with three-state variables. On the other hand, since typically the number of state variables of systems describing elementary circuits is two or three (see [9]), we devote a special attention to the planar case. Here we tackle two problems concerning parameterized families of planar BLCS.

Firstly, obtaining of its stratification diagram with regard to the natural equivalence relation is defined by change of basis in the state space (which preserve the hyperplanes parallel to the separating one). Previously, it is necessary to list the possible equivalence classes and to obtain a complete set of classifying invariant parameters (Theorem 6).
By the way, Arnold's theory allows us to restrict this study to the so-called "miniversal" deformation families. Indeed, the equivalence classes are just the orbits of a certain group action, so that they are differentiable manifolds and Arnold's machinery is applicable. Moreover we remark that by joining the orbits according to the discrete classifying invariants one obtains differentiable "strata" (each one formed by the union of classes differing only on continuous classifying invariants). We list the dimension of each orbit and the corresponding strata (Proposition 7). As an application of the previous results, we present the unobservable bifurcation diagram of a miniversal deformation (Example 10).

Secondly we consider parameterized families of controllable BLCS. It is known (see [3]) that for each value of the parameter there is a feedback which stabilizes the corresponding system. Thus, we lead to the quite general question of whether pointwise solvability implies the existence of a nicely parameterized solution [10]. This parameterized family of pointwise stabilizers may not be differentiable (not even continuous). Our results allow constructing a differentiable family of feedbacks which stabilizes the corresponding system for each value of the parameter (Theorem 15).

We point out that when dealing with parameterized families of BLCS, the nongeneric case of unobservable ones appears in a natural way. See, for example, the circuit modeling the Fitzghugh-Nagumo equations in [9], where the unobservable case appears if $R_{3}=R_{4}$. 
Finally, notice that, as in previous works concerning single control systems, we will use geometrical techniques: reducing bases, stratifications, and miniversal deformations In this sense, we expect that the geometrical approach in [11] could be translated to BLCS in the future.

Throughout the paper, $\mathbb{R}$ will denote the set of real numbers and $M_{n \times m}(\mathbb{R})$ the set of matrices having $n$ rows and $m$ columns and entries in $\mathbb{R}$ (in the case where $n=m$, we will simply write $M_{n}(\mathbb{R})$ ).

\section{Planar Bimodal Linear Control Systems}

Let us consider a bimodal linear control system (BLCS) given by

$$
\begin{gathered}
\dot{x}(t)=A_{1} x(t)+B_{1} u(t), \\
y(t)=C x(t), \\
\dot{x}(t)=A_{2} x(t)+B_{2} u(t), \\
y(t)=C x(t),
\end{gathered}
$$

where $A_{1}, A_{2} \in M_{n}(\mathbb{R}) ; B_{1}, B_{2} \in M_{n \times 1}(\mathbb{R}) ; C \in M_{1 \times n}(\mathbb{R})$. One assumes that the dynamics is continuous along the separating hyperplane $H=\left\{x \in \mathbb{R}^{n}: C x=0\right\}$; that is to say, both subsystems coincide for $y(t)=0$.

By means of a linear change in the state variable $x(t)$, one can consider $C=\left(\begin{array}{llll}1 & 0 & \cdots & 0\end{array}\right) \in M_{1 \times n}(\mathbb{R})$. Hence $H=\{x \in$ $\left.\mathbb{R}^{n}: x_{1}=0\right\}$ and continuity along $H$ is equivalent to

$$
B_{2}=B_{1}, \quad A_{2} e_{i}=A_{1} e_{i}, \quad 2 \leq i \leq n .
$$

We will write from now on $B=B_{1}=B_{2}$.

Definition 1. In the above conditions, one says that the triple of matrices $\left(A_{1}, A_{2}, B\right)$ defines a bimodal linear control system (BLCS). Throughout the paper, $\mathscr{X}$ will denote the set of these triples:

$$
\begin{aligned}
\mathscr{X}=\{ & \left(A_{1}, A_{2}, B\right) \in M_{n}(\mathbb{R}) \times M_{n}(\mathbb{R}) \\
& \left.\times M_{n \times 1}(\mathbb{R}) \mid A_{2} e_{i}=A_{1} e_{i}, 2 \leq i \leq n\right\}
\end{aligned}
$$

which is obviously a $\left(n^{2}+2 n\right)$-differentiable manifold.

The system is called observable if

$$
\operatorname{rank}\left(\begin{array}{c}
C \\
C A_{i} \\
\cdots \\
C A_{i}^{n-1}
\end{array}\right)=n, \quad i=1,2
$$

A natural goal is simplifying the matrices $A_{1}, A_{2}$, and $B$ by means of changes in the variables $x(t)$ which preserve the qualitative behavior of the system. So, one considers linear changes in the state variables space preserving the hyperplanes $x_{1}(t)=k$.
Definition 2. One calls admissible basis changes those given by the matrices

$$
\begin{aligned}
& \mathcal{S}:=\left\{S \in G l_{n}(\mathbb{R}) \mid S=\left(\begin{array}{cc}
1 & 0 \\
U & T
\end{array}\right),\right. \\
&\left.T \in G l_{n-1}(\mathbb{R}), U \in M_{n \times 1}(\mathbb{R})\right\} .
\end{aligned}
$$

Then, $\left(A_{1}, A_{2}, B\right),\left(A_{1}^{\prime}, A_{2}^{\prime}, B^{\prime}\right) \in \mathscr{X}$ are said to be equivalent if there exists a matrix $S \in \mathcal{S}$ (representing an admissible basis change) such that $\left(A_{1}^{\prime}, A_{2}^{\prime}, B^{\prime}\right)=$ $\left(S^{-1} A_{1} S, S^{-1} A_{2} S, S^{-1} B\right)$.

Notice that the matrix $C$ is not involved in this definition since $C S=C$ for any $S \in \mathcal{S}$.

When considering canonical forms, it is necessary that the coefficients appearing in them as well as the conditions used to distinguish the different types do not depend on the admissible basis which one considers; that is to say, they are preserved under admissible basis changes $S \in \mathcal{S}$. It is wellknown that $\operatorname{tr} A_{1}, \operatorname{tr} A_{2}$, $\operatorname{det} A_{1}$, and $\operatorname{det} A_{2}$ are invariant under any basis change $S \in G l_{n}(\mathbb{R})$. We focus on the additional invariants when only admissible basis changes $S \in$ $\mathcal{S}$ are considered.

Definition 3. A real number (resp., a property) associated with a triple $\left(A_{1}, A_{2}, B\right)$ is called $\mathcal{S}$-invariant if it is preserved by admissible basis changes; that is to say, it has the same value (resp., it is also true) for any other triple $\left(A_{1}^{\prime}, A_{2}^{\prime}, B^{\prime}\right) \mathcal{S}$ equivalent to the given one.

For example, it is obvious that they are $S$-invariant: the top coefficient $b_{1}$ in $B$, the matrix $C$, and the condition of $\left(A_{1}, A_{2}, B\right)$ being observable.

We introduce another $\mathcal{S}$-invariant that will be used under additional hypotheses.

Definition 4. Given a triple

$$
A_{1}=\left(\begin{array}{ll}
a_{1} & a_{3} \\
a_{2} & a_{4}
\end{array}\right), \quad A_{2}=\left(\begin{array}{ll}
\gamma_{1} & a_{3} \\
\gamma_{2} & a_{4}
\end{array}\right), \quad B=\left(\begin{array}{l}
b_{1} \\
b_{2}
\end{array}\right)
$$

one writes

$$
\begin{gathered}
\Delta_{0}=\operatorname{det}\left(\begin{array}{ll}
a_{3} & b_{1} \\
a_{4} & b_{2}
\end{array}\right)=a_{3} b_{2}-a_{4} b_{1}, \\
\Delta_{12}=a_{2}\left(a_{4}-\gamma_{1}\right)-\gamma_{2}\left(a_{4}-a_{1}\right), \\
\Delta_{1}=b_{1} a_{2}+\left(a_{4}-a_{1}\right) b_{2}, \\
\Delta_{2}=b_{1} \gamma_{2}+\left(a_{4}-\gamma_{1}\right) b_{2} .
\end{gathered}
$$

Lemma 5. The above triple is unobservable if and only if $a_{3}=$ 0 . In this case one has

$$
\begin{gathered}
\text { (1) } \operatorname{det}\left(\left(\begin{array}{c}
a_{1}-\gamma_{1} \\
a_{2}-\gamma_{2}
\end{array}\right) \mid A_{i}\left(\begin{array}{c}
a_{1}-\gamma_{1} \\
a_{2}-\gamma_{2}
\end{array}\right)\right)=\left(a_{1}-\gamma_{1}\right) \Delta_{12}, i=1,2, \\
\operatorname{det}\left(B \mid A_{i} B\right)=b_{1} \Delta_{i}, i=1,2 ;
\end{gathered}
$$


(2) the action of $S \in \mathcal{S}$ transforms $\Delta_{1}, \Delta_{2}$, and $\Delta_{12}$, respectively, into

$$
\frac{1}{\operatorname{det} S} \Delta_{1}, \quad \frac{1}{\operatorname{det} S} \Delta_{2}, \quad \frac{1}{\operatorname{det} S} \Delta_{12} .
$$

In particular, it is $\mathcal{S}$-invariant the sign (positive, negative, or zero):

$$
\operatorname{sign}\left(\Delta_{1} \Delta_{2}\right)
$$

Proof. Clearly,

$$
\left(\begin{array}{c}
C \\
C A_{1}
\end{array}\right)=\left(\begin{array}{cc}
1 & 0 \\
a_{1} & a_{3}
\end{array}\right), \quad\left(\begin{array}{c}
C \\
C A_{2}
\end{array}\right)=\left(\begin{array}{cc}
1 & 0 \\
\gamma_{1} & a_{3}
\end{array}\right)
$$

do not have maximal rank when $a_{3}=0$. Then

(1) it is a straightforward computation;

(2) if $a_{3}=0$, then $a_{1}$ and $\gamma_{1}$ are eigenvalues of $A_{1}$ and $A_{2}$.

The action of $S$ transforms the matrices in $(*)$ into their left product by $S^{-1}$.

Theorem 6. With the above notation:

(1) Table 1 summarizes some $\mathcal{S}$-invariant numbers and properties, as well as the hypotheses for each one;

(2) Table 2 lists the possible canonical forms and the classification criteria.

\section{Proof. (i) Concerning Table 1}

(1) the $\mathcal{S}$-action on $A_{1}$ and $B$ can be formulated as

$$
\begin{aligned}
& S^{-1}\left(A_{1}, B\right)\left(\frac{S \mid 0}{0 \mid 1}\right) \\
& =\left(\begin{array}{ll}
1 & 0 \\
u & t
\end{array}\right)^{-1}\left(\begin{array}{lll}
a_{1} & a_{3} & b_{1} \\
a_{2} & a_{4} & b_{2}
\end{array}\right)\left(\begin{array}{lll}
1 & 0 & 0 \\
u & t & 0 \\
0 & 0 & 1
\end{array}\right) \\
& =\left(\begin{array}{l}
* \\
*
\end{array} \mid\left(\begin{array}{ll}
1 & 0 \\
u & t
\end{array}\right)^{-1}\left(\begin{array}{ll}
a_{3} & b_{1} \\
a_{4} & b_{2}
\end{array}\right)\left(\begin{array}{ll}
t & 0 \\
0 & 1
\end{array}\right)\right) .
\end{aligned}
$$

Therefore, $\Delta_{0}$ is $\mathcal{S}$-invariant:

$$
\operatorname{det}\left(\left(\begin{array}{ll}
1 & 0 \\
u & t
\end{array}\right)^{-1}\left(\begin{array}{ll}
a_{3} & b_{1} \\
a_{4} & b_{2}
\end{array}\right)\left(\begin{array}{ll}
t & 0 \\
0 & 1
\end{array}\right)\right)=\operatorname{det}\left(\begin{array}{ll}
a_{3} & b_{1} \\
a_{4} & b_{2}
\end{array}\right) .
$$

We have seen that $a_{3} \neq 0$ if and only if

$$
\operatorname{rank}\left(\begin{array}{c}
C \\
C A_{i}
\end{array}\right)=2, \quad i=1,2,
$$

which is $\mathcal{S}$-invariant.
(2) If $b_{1}=0$, then

$$
S^{-1}\left(\begin{array}{c}
0 \\
b_{2}
\end{array}\right)=\frac{1}{t}\left(\begin{array}{cc}
t & 0 \\
-u & 1
\end{array}\right)\left(\begin{array}{c}
0 \\
b_{2}
\end{array}\right)=\frac{1}{t}\left(\begin{array}{c}
0 \\
b_{2}
\end{array}\right) .
$$

(3) If $a_{3}=0$, then $a_{1}, a_{4}$, and $\gamma_{1}$ are the eigenvalues of $A_{1}$ and $A_{2}$. Then

$$
\operatorname{Ker}\left(A_{1}-a_{1} I\right)=\operatorname{Ker}\left(A_{2}-\gamma_{1} I\right)
$$

if and only if

$$
\operatorname{rank}\left(\begin{array}{cc}
a_{2} & a_{4}-a_{1} \\
\gamma_{2} & a_{4}-\gamma_{1}
\end{array}\right)=1
$$

or, equivalently,

$$
0=\operatorname{det}\left(\begin{array}{ll}
a_{2} & a_{4}-a_{1} \\
\gamma_{2} & a_{4}-\gamma_{1}
\end{array}\right)=\Delta_{12}
$$

In a similar way, $\Delta_{1}=0$ if and only if

$$
\left(\begin{array}{l}
b_{1} \\
b_{2}
\end{array}\right) \in \operatorname{Ker}\left(A_{1}-a_{1} I\right)
$$

and $\Delta_{2}=0$ if and only if

$$
\left(\begin{array}{l}
b_{1} \\
b_{2}
\end{array}\right) \in \operatorname{Ker}\left(A_{2}-\gamma_{1} I\right)
$$

$\left(3^{\prime}\right)$ This case follows from (3) and the above lemma.

(4) Clearly, if $a_{3}=0$ and $a_{1}=a_{4}$, then $a_{2}=0$ if and only if $A_{1}$ diagonalizes.

$\left(4^{\prime}\right)$ Analogously than (4) for $\gamma_{2}=0$.

(5) Returning to the formulation in (1):

$$
\left(\begin{array}{ll}
1 & 0 \\
u & t
\end{array}\right)^{-1}\left(\begin{array}{ccc}
a_{1} & 0 & 0 \\
a_{2} & a_{1} & b_{2}
\end{array}\right)\left(\begin{array}{lll}
1 & 0 & 0 \\
u & t & 0 \\
0 & 0 & 1
\end{array}\right)=\left(\begin{array}{ccc}
a_{1} & 0 & 0 \\
\frac{a_{2}}{t} & a_{1} & \frac{b_{2}}{t}
\end{array}\right) .
$$

$\left(5^{\prime}\right)$ Analogously than (5) for $\gamma_{1}=a_{4}$.

(ii) This case follows from [12], bearing in mind the $\mathcal{S}$ invariants in (i).

\section{Stratification Diagrams}

In the previous section we have partitioned the set of BLCS into equivalence classes, characterized by the reduced forms in Theorem 6. In order to study, for example, the changes when a BLCS is perturbed, some natural questions arise about the geometric structure of this equivalence partition. We will see in a moment that each equivalence class is a manifold, as well as the "strata" obtained by joining the classes that differ only in the continuous classification parameters. Their dimensions are listed in Proposition 7.

Concerning perturbations, small changes in the coefficients of the matrices defining the system may give rise to 
TABLE 1: $\mathcal{S}$-Invariant numbers.

\begin{tabular}{|c|c|c|c|}
\hline & Hypotheses & Numbers & Properties \\
\hline (1) & & $\Delta_{0}, b_{1}$ & $a_{3}=0$ \\
\hline (2) & $b_{1}=0$ & & $b_{2}=0$ \\
\hline (3) & $a_{3}=0$ & $a_{1}, \gamma_{1}, a_{4}$ & $\Delta_{12}=0, \Delta_{1}=0, \Delta_{2}=0$ \\
\hline$\left(3^{\prime}\right)$ & $a_{3}=0, \Delta_{12} \neq 0$ & $\Delta_{1} / \Delta_{12}, \Delta_{2} / \Delta_{12}$ & \\
\hline (4) & $a_{3}=0, a_{1}=a_{4}$ & & $a_{2}=0$ \\
\hline$\left(4^{\prime}\right)$ & $a_{3}=0, \gamma_{1}=a_{4}$ & & $\gamma_{2}=0$ \\
\hline (5) & $b_{1}=0, a_{3}=0, a_{1}=a_{4}$ & $b_{2} / a_{2}$ & \\
\hline$\left(5^{\prime}\right)$ & $b_{1}=0, a_{3}=0, \gamma_{1}=a_{4}$ & $b_{2} / \gamma_{2}$ & \\
\hline
\end{tabular}

TABLE 2: Canonical forms.

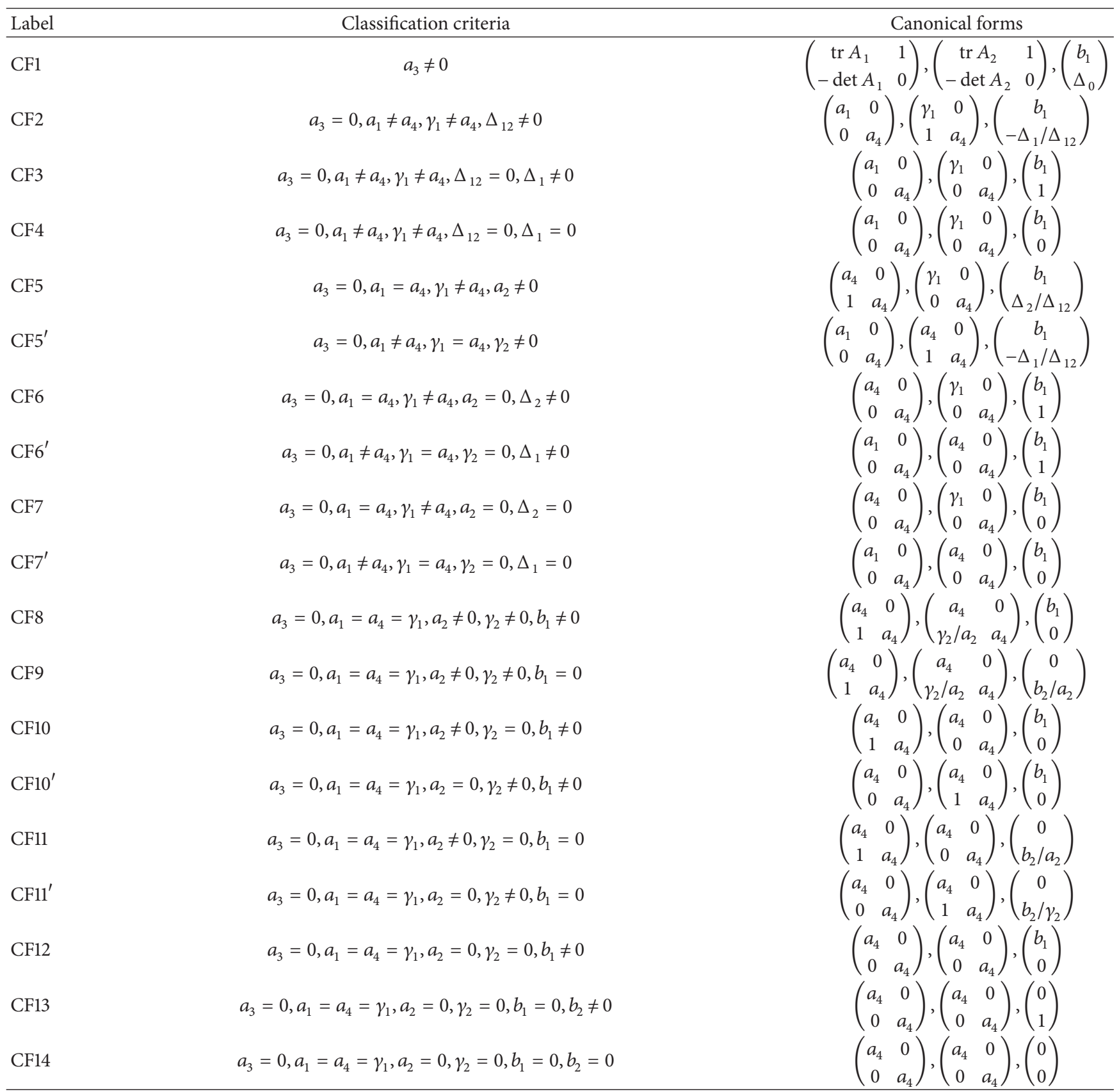


TABLE 3: Dimension of orbits and strata for each case in Table 2.

\begin{tabular}{lcc}
\hline Canonical form & $\begin{array}{c}\text { Dimension of } \\
\text { the orbit }\end{array}$ & $\begin{array}{c}\text { Dimension of } \\
\text { the stratum }\end{array}$ \\
\hline CF1 & 2 & 8 \\
CF2 & 2 & 7 \\
CF3 & 2 & 6 \\
CF4 & 1 & 5 \\
CF5, CF5' & 2 & 6 \\
CF6, CF6' & 2 & 5 \\
CF7, CF7' & 1 & 4 \\
CF8 & 2 & 5 \\
CF9 & 1 & 4 \\
CF10, CF10 & 2 & 4 \\
CF11, CF11 & 1 & 3 \\
CF12 & 1 & 3 \\
CF13 & 1 & 2 \\
CF14 & 0 & 1 \\
\hline
\end{tabular}

nonequivalent systems. Then in order to explain the behavior of the system under small perturbations, it is necessary to know the nearby equivalence classes. The "stratification diagram" ("bifurcation diagram" in Arnold's terminology) of a parameterized family of systems is the partition of the parameter space according to the equivalence class. Arnold's theory [13] shows that they are induced by the ones of the so-called versal deformations. In particular, the miniversal deformations are the simplest parameterized families which provide all the information about which equivalence classes are near a given one, that is to say, which canonical forms appear when the given one is perturbed.

The starting point is that the above equivalence classes are actually the orbits with regard to the action of the Lie group $\mathcal{S}$ on the differentiable manifold $\mathscr{X}$ :

$$
\alpha: \mathcal{S} \times \mathscr{X} \longrightarrow \mathscr{X}
$$

defined by

$$
\alpha(S, \mathscr{X})=\left(S^{-1} A_{1} S, S^{-1} A_{2} S, S^{-1} B\right) .
$$

Given any triple of matrices $\left(A_{1}, A_{2}, B\right) \in \mathscr{X}$, we will denote by $\mathcal{O}\left(A_{1}, A_{2}, B\right)$ its orbit (or equivalence class).

As an application of the closed orbit lemma (see [14]), we deduce that equivalence classes are differentiable manifolds. Namely, any equivalence class is a locally closed differentiable submanifold of $\mathscr{X}$ and its boundary is a union of equivalence classes or orbits of strictly lower dimension. In particular, equivalence classes or orbits of minimal dimension are closed.

Moreover, orbits having the same discrete classification parameters (but differing in the continuous ones) can be joined in a finite number of "strata" which in our case are as well differentiable manifolds (see [12]). For the commodity of the reader we adapt the results there.

Proposition 7. Table 3 lists the dimensions of the orbits (i.e., the equivalence classes) and the corresponding strata (i.e., the union of the orbits of the same type when the parameters appearing in the canonical form vary).

To illustrate the above considerations and as an application of the results in Section 2, we will present (see Figure 1) the unobservable bifurcation diagram of the miniversal deformation of a system of type CF10'. The main definitions and results about deformations and versality can be found in $[13,15]$. Here we rewrite them down, adapted to our particular case.

Definition 8. A deformation of $\left(A_{1}, A_{2}, B\right) \in \mathscr{X}$ is a differentiable map $\varphi: U \rightarrow \mathscr{X}$, with $U$ an open neighbourhood of the origin $\mathbb{R}^{d}$, such that $\varphi(0)=\left(A_{1}, A_{2}, B\right)$.

A deformation $\varphi: U \rightarrow \mathscr{X}$ of $\left(A_{1}, A_{2}, B\right)$ is called versal at 0 if, for any other deformation of $\left(A_{1}, A_{2}, B\right), \psi$ : $V \rightarrow \mathscr{X}$, there exists a neighbourhood $V^{\prime} \subseteq V$ with $0 \in V^{\prime}$, a differentiable map $\gamma: V^{\prime} \rightarrow U$ with $\gamma(0)=0$, and a deformation of the identity $I \in \mathcal{S}, \theta: V^{\prime} \rightarrow \mathcal{S}$, such that $\psi(\mu)=\alpha(\theta(\mu), \varphi(\gamma(\mu)))$ for all $\mu \in V^{\prime}$.

A versal deformation with minimal number of parameters $d$ is called miniversal deformation.

A miniversal deformation can be obtained from the normal space to the orbit with regard to some scalar product.

Proposition 9 (see [16]). We consider the following scalar product in $X$ :

$$
\begin{aligned}
& \left\langle\left(A_{1}, A_{2}, B\right),\left(A_{1}^{\prime}, A_{2}^{\prime}, B^{\prime}\right)\right\rangle \\
& \quad=\operatorname{tr}\left(A_{1}^{t} A_{1}^{\prime}\right)+\operatorname{tr}\left(A_{2}^{t} A_{2}^{\prime}\right)+\operatorname{tr}\left(B^{t} B^{\prime}\right) .
\end{aligned}
$$

(i) The normal space to the orbit of $\left(A_{1}, A_{2}, B\right)$ at $\left(A_{1}, A_{2}\right.$, $B) N_{\left(A_{1}, A_{2}, B\right)} \mathcal{O}\left(A_{1}, A_{2}, B\right) \cap \mathscr{X}$ is the vector subspace consisting of triples $\left(X_{1}, X_{2}, Y\right) \in \mathscr{X}$ such that

$$
A_{1} X_{1}^{t}-X_{1}^{t} A_{1}+A_{2} X_{2}^{t}-X_{2}^{t} A_{2}-B Y^{t} \in \mathbb{A},
$$

where $\mathbb{A}$ is the set

$$
\mathbb{A}=\left\{M=\left(m_{i}^{j}\right) \mid m_{i}^{j}=0,2 \leq i \leq n, 1 \leq j \leq n\right\} .
$$

(ii) Then the mapping

$$
\begin{gathered}
\mathbb{R}^{d} \longrightarrow x \\
\left(\eta_{1}, \ldots, \eta_{d}\right) \longrightarrow\left(A_{1}, A_{2}, B\right)+\eta_{1} V_{1}+\cdots+\eta_{d} V_{d},
\end{gathered}
$$

where $\left\{V_{1}, \ldots, V_{d}\right\}$ is any basis of the vector space $N_{\left(A_{1}, A_{2}, B\right)} \mathcal{O}\left(A_{1}, A_{2}, B\right)$, is a miniversal deformation of $\left(A_{1}, A_{2}, B\right)$.

Normal spaces of two equivalent triples can be obtained one from the other. Thus, it is always possible to restrict ourselves to the case where the triple is in its canonical form.

Here, as an application of the previous results, we present the unobservable bifurcation diagram of a miniversal deformation: Figure 1 shows the geometrical configuration of the unobservable strata near a given system of type CF10'. 
Example 10. Consider a bimodal linear dynamical system of type $\mathrm{CF} 10^{\prime}$ whose canonical form is

$$
A_{1}=\left(\begin{array}{cc}
a_{4} & 0 \\
0 & a_{4}
\end{array}\right), \quad A_{2}=\left(\begin{array}{cc}
a_{4} & 0 \\
1 & a_{4}
\end{array}\right), \quad B=\left(\begin{array}{c}
b_{1} \\
0
\end{array}\right) .
$$

Then, $N_{\left(A_{1}, A_{2}, B\right)} \mathcal{O}\left(A_{1}, A_{2}, B\right) \cap \mathscr{X}$ is the vector subspace consisting of triples $\left(X_{1}, X_{2}, Y\right) \in \mathscr{X}$

$$
X_{1}=\left(\begin{array}{ll}
x_{1} & x_{3} \\
x_{2} & x_{4}
\end{array}\right), \quad X_{2}=\left(\begin{array}{ll}
x_{5} & x_{3} \\
x_{6} & x_{4}
\end{array}\right), \quad Y=\left(\begin{array}{l}
y_{1} \\
y_{2}
\end{array}\right)
$$

such that

$$
\begin{gathered}
x_{6}=0 \\
a_{4} x_{5}+b_{1} y_{2}=0 .
\end{gathered}
$$

Moreover, parameter $x_{3}$ must be zero to avoid observable perturbations and parameters $x_{4}, y_{1}$ give orbits in the initial stratum.

Then the unobservable perturbations in the normal space to the stratum of $\left(A_{1}, A_{2}, B\right)$ are parameterized by

$$
\begin{aligned}
\varphi & \left(x_{1}, x_{2}, x_{5}\right) \\
& =\left(\left(\begin{array}{cc}
a_{4}+x_{1} & 0 \\
x_{2} & a_{4}
\end{array}\right),\left(\begin{array}{cc}
a_{4}+x_{5} & 0 \\
1 & a_{4}
\end{array}\right),\left(\begin{array}{c}
b_{1} \\
-\frac{a_{4}}{b_{1}} x_{5}
\end{array}\right)\right) .
\end{aligned}
$$

We denote by $E_{i}$ the set of all triples of matrices having canonical form of type (CFi), $i=1, \ldots, 14$.

Clearly, if only $x_{1}$ (resp., $x_{2}$ ) is nonzero, it lies in $E 5^{\prime}$ (resp., E8). But for only $x_{5}$, the strata E6 and E7 are possible in principle, depending on the value of $\Delta_{2}$. In our case

$$
\begin{aligned}
\Delta_{2} & =b_{1} \gamma_{2}+\left(a_{4}-\gamma_{1}\right) b_{2}=b_{1}+\left(-x_{5}\right)\left(-\frac{a_{4}}{b_{1}} x_{5}\right) \\
& =\frac{1}{b_{1}}\left(b_{1}^{2}+a_{4} x_{5}^{2}\right) .
\end{aligned}
$$

Hence, it belongs to E7 for $x_{5}^{2}=-b_{1}^{2} / a_{4}$, and to E6 otherwise.

In a similar way, if $x_{1}, x_{5} \neq 0$ only E2, E3, and E4 are possible. We have $\Delta_{0}=-x_{2} x_{5}+x_{1}$. Hence, $x_{2}=0$ implies $\Delta_{0} \neq 0$, which corresponds to E2. If $x_{2} \neq 0$, it gives again E2 except on the hyperbolic paraboloid $x_{1}=x_{2} x_{5}$. When it happens,

$$
\Delta_{1}=b_{1} x_{2}+x_{1} \frac{a_{4}}{b_{1}} x_{5}=\frac{x_{2}}{b_{1}}\left(b_{1}^{2}+a_{4} x_{5}^{2}\right) .
$$

Hence, it lies in E4 for $x_{5}^{2}=-b_{1}^{2} / a_{4}$, and in $E 3$ otherwise.

Finally, it is straightforward that one obtains E5 for $x_{1}=$ $0, x_{2}, x_{5} \neq 0$, and $E 5^{\prime}$ for $x_{5}=0, x_{1}, x_{2} \neq 0$. In summary (see Figure 1),

(i) if $x_{2}, x_{5}=0, x_{1} \neq 0$, then $\varphi\left(x_{1}, x_{2}, x_{5}\right) \in E 5^{\prime}$;

(ii) if $x_{1}, x_{5}=0, x_{2} \neq 0$, then $\varphi\left(x_{1}, x_{2}, x_{5}\right) \in E 8$;

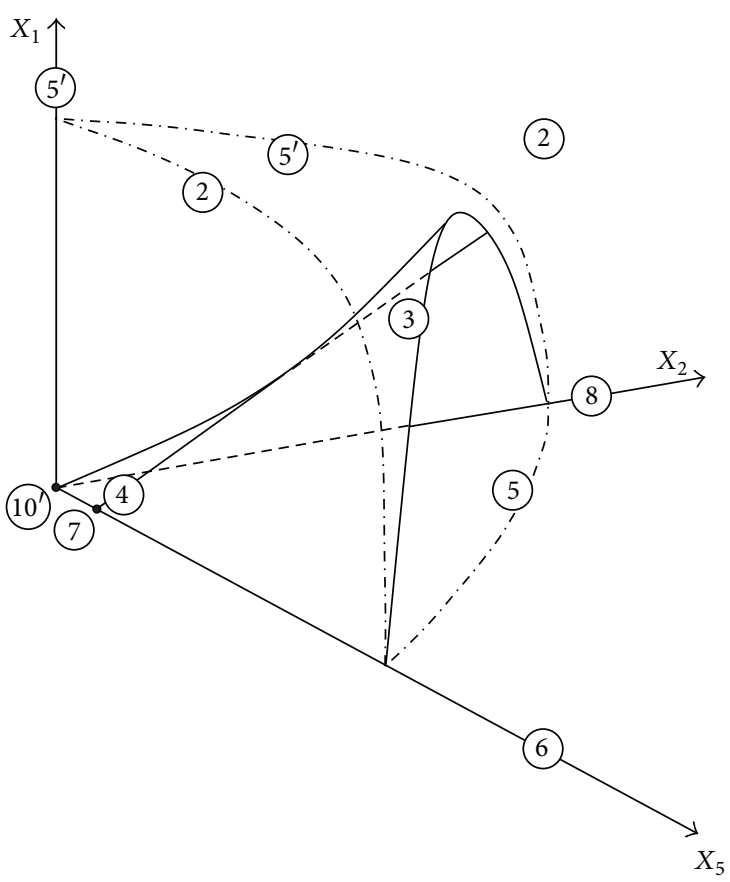

FIGURE 1: Stratification diagram.

(iii) if $x_{1}, x_{2}=0, x_{5}^{2}=-b_{1}^{2} / a_{4}$, then $\varphi\left(x_{1}, x_{2}, x_{5}\right) \in E 7$;

(iv) if $x_{1}, x_{2}=0, x_{5} \neq 0, x_{5}^{2} \neq-b_{1}^{2} / a_{4}$, then $\varphi\left(x_{1}, x_{2}, x_{5}\right) \in$ E6;

(v) if $x_{5}=0, x_{1}, x_{2} \neq 0$, then $\varphi\left(x_{1}, x_{2}, x_{5}\right) \in E 5^{\prime}$;

(vi) if $x_{2}=0, x_{1}, x_{5} \neq 0$, then $\varphi\left(x_{1}, x_{2}, x_{5}\right) \in E 2$;

(vii) if $x_{1}=0, x_{2}, x_{5} \neq 0$, then $\varphi\left(x_{1}, x_{2}, x_{5}\right) \in E 5$;

(viii) if $x_{1}, x_{2}, x_{5} \neq 0, x_{1}=x_{2} x_{5}, x_{5}^{2}=-b_{1}^{2} / a_{4}$, then $\varphi\left(x_{1}, x_{2}, x_{5}\right) \in E 4$

(ix) if $x_{1}, x_{2}, x_{5} \neq 0, x_{1}=x_{2} x_{5}, x_{5}^{2} \neq-b_{1}^{2} / a_{4}$, then $\varphi\left(x_{1}, x_{2}, x_{5}\right) \in E 3 ;$

(x) if $x_{1}, x_{2}, x_{5} \neq 0, x_{1} \neq x_{2} x_{5}$, then $\varphi\left(x_{1}, x_{2}, x_{5}\right) \in E 2$.

\section{Controllability and Families of Stabilizers}

We have seen that in a differentiable family of BLCS different equivalence classes can appear for different values of the parameters. Let us see that, however, some global treatments are possible. Indeed, we will prove that a differentiable family of stabilizers exists if each BLCS in the given family is controllable.

The notion of controllability of a single system is extended to bimodal ones in a natural way.

Definition 11. A BLCS is (completely) controllable if for any pair of states $\left(x_{0}, x_{f}\right)$ there exists a locally integrable input $u$ such that the solution $x^{x_{0}, u}$ passes through $x_{f}$; that is, $x^{x_{0}, u}(T)=x_{f}$ for some $T>0$. 
A well-known remarkable fact is that a single linear system $\dot{x}=A x+B u$ is controllable if and only if its "controllability matrix" ( $\left.B \quad A B \cdots A^{n-1} B\right)$ has maximal rank. For planar BLCS we recall the characterization of controllability of planar BLCS obtained in [1] for observable systems and generalized in [17] to unobservable ones.

Proposition 12. Let one consider a planar BLCS defined by $\left(A_{1}, A_{2}, B\right)$. One writes $C_{1}, C_{2}$ the controllability matrices of both subsystems

$$
C_{1}=\left(\begin{array}{ll}
B & A_{1} B
\end{array}\right), \quad C_{2}=\left(\begin{array}{ll}
B & A_{2} B
\end{array}\right) .
$$

Then, it is controllable if and only if

$$
\operatorname{det} C_{1} \operatorname{det} C_{2}>0 \text {. }
$$

Remark 13. (1) Notice that, in particular, both subsystems must be controllable, but it is not a sufficient condition.

(2) Whereas for single systems the subset of controllable ones is open and dense, the above proposition shows that it is not for BLCS systems: controllability is an open, but not generic, property.

If the control function is a so-called "feedback" of the type $u(t)=f(x(t))$, one obtains a new dynamical system ("in closed loop"). For single linear systems $\dot{x}=A x+B u$, a feedback $u=F x$ gives $\dot{x}=(A+B F) x$. A remarkable fact is that it is stable for some suitable $F$, provided that the initial control system is controllable.

As a natural generalization, in [3] any controllable BLCS is proved to be feedback stabilizable. Hence, if a differentiable parameterized family $\left(A_{1}(s), A_{2}(s), B(s)\right)$ is pointwise controllable (observable or not), then it is also pointwise stabilizable; that is to say, for any $s \in \mathbb{R}$ there is a common feedback $F(s)$ such that both closed-loop systems $A_{1}(s)+$ $B(s) F(s), A_{2}(s)+B(s) F(s)$ are stable. However, the family $F(s)$ may not be differentiable (not even continuous). Here we prove that differentiable families of stabilizer feedbacks exist for $n=2$.

As we have pointed out in the Introduction, the unobservable case appears generically in parameterized families of bimodal systems. A typical case is considered in the following example. As an application of the above proposition, we characterize when this family is pointwise controllable.

Example 14. Let us consider the parameterized family of planar BLCS

$$
A_{1}(s)=\left(\begin{array}{cc}
a_{1} & s \\
a_{2} & a_{4}
\end{array}\right), \quad A_{2}(s)=\left(\begin{array}{cc}
\gamma_{1} & s \\
\gamma_{2} & a_{4}
\end{array}\right), \quad B=\left(\begin{array}{l}
b_{1} \\
b_{2}
\end{array}\right),
$$

where $s \in \mathbb{R}$. Obviously, the systems defined by these matrices are observable except for $s=0$. Let us see that the family is pointwise controllable (i.e., for any $s \in \mathbb{R}$ the corresponding system is controllable) if and only if $b_{1} \neq 0$ and

(i) $a_{2} \gamma_{2}>0$, if $b_{2}=0$,

(ii) $\operatorname{det}\left(\begin{array}{ll}a_{1} & b_{1} \\ a_{2} & b_{2}\end{array}\right)=\operatorname{det}\left(\begin{array}{ll}\gamma_{1} & b_{1} \\ \gamma_{2} & b_{2}\end{array}\right)$, otherwise.
From Proposition 12, for any $s \in \mathbb{R}$ (including the case where $s=0$ ) the corresponding system is controllable if and only if

$$
\begin{aligned}
\left(b_{1}^{2} a_{2}\right. & \left.+b_{1} b_{2} a_{4}-b_{1} b_{2} a_{1}-b_{2}^{2} s\right) \\
& \times\left(b_{1}^{2} \gamma_{2}+b_{1} b_{2} a_{4}-b_{1} b_{2} \gamma_{1}-b_{2}^{2} s\right)>0 .
\end{aligned}
$$

In particular $b_{1} \neq 0$ (it suffices to take $s=0$ ).

If $b_{2}=0$, the above inequality is

$$
\left(b_{1}^{2} a_{2}\right)\left(b_{1}^{2} \gamma_{2}\right)>0
$$

that is to say,

$$
a_{2} \gamma_{2}>0
$$

Assume now $b_{2} \neq 0$. In general, two polynomials of degree 1 have the same sign at any point if and only if they have the same root and the slopes have the same sign. In our case both slopes are $-b_{2}^{2}$, so that the above inequality holds if and only if

$$
\frac{b_{1}^{2} a_{2}+b_{1} b_{2} a_{4}-b_{1} b_{2} a_{1}}{b_{2}^{2}}=\frac{b_{1}^{2} \gamma_{2}+b_{1} b_{2} a_{4}-b_{1} b_{2} \gamma_{1}}{b_{2}^{2}}
$$

which is equivalent (recall $b_{1} \neq 0$ ) to

$$
b_{1} a_{2}-b_{2} a_{1}=b_{1} \gamma_{2}-b_{2} \gamma_{1} \text {. }
$$

Finally, we prove the existence of differentiable families of stabilizers for differentiable families of planar controllable bimodal systems.

Theorem 15. Let

$$
\left(A_{1}(s), A_{2}(s), B(s)\right), \quad s \in \mathbb{R}
$$

be a differentiable family of planar BLCS. If it is pointwise controllable, then there is a differentiable family of feedbacks $F(s), s \in \mathbb{R}$, such that

$$
A_{1}(s)+B(s) F(s), \quad A_{2}(s)+B(s) F(s)
$$

are stable for any $s \in \mathbb{R}$.

More explicitly, if

$$
\begin{gathered}
A_{1}(s)=\left(\begin{array}{ll}
a_{1} & a_{3} \\
a_{2} & a_{4}
\end{array}\right), \quad A_{2}(s)=\left(\begin{array}{ll}
\gamma_{1} & a_{3} \\
\gamma_{2} & a_{4}
\end{array}\right), \\
B(s)=\left(\begin{array}{l}
b_{1} \\
b_{2}
\end{array}\right), \\
C_{1}=\left(B(s) \quad A_{1}(s) B(s)\right), \quad C_{2}=\left(B(s) A_{2}(s) B(s)\right),
\end{gathered}
$$

where all the coefficients are assumed to be differentiably depending on $s \in \mathbb{R}$, one can take

$$
\begin{aligned}
F & =\left(\begin{array}{ll}
f_{1} & f_{2}
\end{array}\right) \\
& =\left(\begin{array}{ll}
x & y
\end{array}\right) \frac{1}{\operatorname{det} C_{1}}\left(\begin{array}{cc}
\operatorname{det}\left(\begin{array}{ll}
b_{1} & a_{1} \\
b_{2} & a_{2}
\end{array}\right) \operatorname{det}\left(\begin{array}{ll}
b_{1} & a_{3} \\
-b_{2} & a_{4}
\end{array}\right) \\
-b_{1}
\end{array}\right)
\end{aligned}
$$


with

$$
\begin{gathered}
x<-\operatorname{tr} A_{1},-\operatorname{tr} A_{2}, \\
y<\operatorname{det} A_{1}, \\
y<-\operatorname{det}\left(\begin{array}{ll}
b_{1} & \gamma_{1}-a_{1} \\
b_{2} & \gamma_{2}-a_{2}
\end{array}\right) \operatorname{det}\left(\begin{array}{ll}
b_{1} & a_{3} \\
b_{2} & a_{4}
\end{array}\right) \frac{1}{\operatorname{det} C_{2}} x \\
+\operatorname{det} A_{2} \frac{\operatorname{det} C_{1}}{\operatorname{det} C_{2}} .
\end{gathered}
$$

Proof. By hypothesis, we assume

$$
\operatorname{det} C_{1} \operatorname{det} C_{2}>0
$$

for any $s \in \mathbb{R}$.

We look for $F(s)=\left(\begin{array}{ll}f_{1} & f_{2}\end{array}\right)$ where again we assume the coefficients depending on $s \in \mathbb{R}$, such that the eigenvalues of

$$
\left(\begin{array}{ll}
a_{1}+b_{1} f_{1} & a_{3}+b_{1} f_{2} \\
a_{2}+b_{2} f_{1} & a_{4}+b_{2} f_{2}
\end{array}\right), \quad\left(\begin{array}{ll}
\gamma_{1}+b_{1} f_{1} & a_{3}+b_{1} f_{2} \\
\gamma_{2}+b_{2} f_{1} & a_{4}+b_{2} f_{2}
\end{array}\right)
$$

have negative real part for any $s \in \mathbb{R}$ or, equivalently, the matrices have negative trace and positive determinant; that is to say,

$$
\begin{gathered}
b_{1} f_{1}+b_{2} f_{2}<-a_{1}-a_{4}, \\
b_{1} f_{1}+b_{2} f_{2}<-\gamma_{1}-a_{4}, \\
f_{1}\left(a_{3} b_{2}-a_{4} b_{1}\right)+f_{2}\left(a_{2} b_{1}-a_{1} b_{2}\right)<a_{1} a_{4}-a_{2} a_{3}, \\
f_{1}\left(a_{3} b_{2}-a_{4} b_{1}\right)+f_{2}\left(\gamma_{2} b_{1}-\gamma_{1} b_{2}\right)<\gamma_{1} a_{4}-\gamma_{2} a_{3} .
\end{gathered}
$$

We change the variables $\left(f_{1}, f_{2}\right)$ by $(x, y)$ defined by

$$
\begin{gathered}
x=b_{1} f_{1}+b_{2} f_{2}, \\
y=\left(b_{2} a_{3}-b_{1} a_{4}\right) f_{1}+\left(b_{1} a_{2}-b_{2} a_{1}\right) f_{2},
\end{gathered}
$$

which is a change of variables, because (by hypothesis)

$$
\operatorname{det}\left(\begin{array}{ll}
b_{1} & b_{2} a_{3}-b_{1} a_{4} \\
b_{2} & b_{1} a_{2}-b_{2} a_{1}
\end{array}\right)=\operatorname{det} C_{1} \neq 0
$$

Then

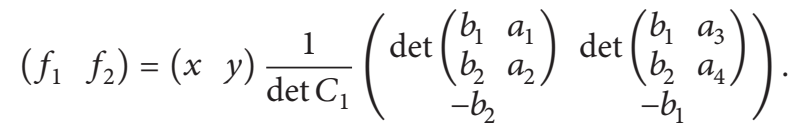

With this change of variables, the desired inequalities become

$$
\begin{gathered}
x<-a_{1}-a_{4}=-\operatorname{tr} A_{1}, \\
x<-\gamma_{1}-a_{4}=-\operatorname{tr} A_{2}, \\
y<a_{1} a_{4}-a_{2} a_{3}=\operatorname{det} A_{1}, \\
\left(a_{3} b_{2}-a_{4} b_{1}\right) \frac{\left(b_{1} a_{2}-b_{2} a_{1}\right) x-b_{2} y}{\operatorname{det} C_{1}} \\
-\left(\gamma_{2} b_{1}-\gamma_{1} b_{2}\right) \frac{\left(b_{2} a_{3}-b_{1} a_{4}\right) x-b_{1} y}{\operatorname{det} C_{1}} \\
<\gamma_{1} a_{4}-\gamma_{2} a_{3} .
\end{gathered}
$$

It is straightforward that the last inequality can be rewritten:

$$
\operatorname{det}\left(\begin{array}{ll}
b_{1} & \gamma_{1}-a_{1} \\
b_{2} & \gamma_{2}-a_{2}
\end{array}\right) \operatorname{det}\left(\begin{array}{ll}
b_{1} & a_{3} \\
b_{2} & a_{4}
\end{array}\right) \frac{1}{\operatorname{det} C_{1}} x+y \frac{\operatorname{det} C_{2}}{\operatorname{det} C_{1}}<\operatorname{det} A_{2} \text {. }
$$

Example 16. For the family in Example 14, when $b_{1} \neq 0, b_{2}=0$, $a_{2} \gamma_{2}>0$, differentiable families of feedbacks are given by

$$
\begin{gathered}
\left(\begin{array}{cc}
f_{1} & f_{2}
\end{array}\right)=\left(\begin{array}{ll}
x & y
\end{array}\right) \frac{1}{b_{1} a_{2}}\left(\begin{array}{cc}
a_{2} & -a_{4} \\
0 & -1
\end{array}\right), \\
x<-a_{1}-a_{4},-\gamma_{1}-a_{4}, \\
y<a_{1} a_{4}-a_{2} s, \\
y<\left(\frac{a_{2}}{\gamma_{2}}-1\right) a_{4} x+\frac{a_{2}}{\gamma_{2}} \gamma_{1} a_{4}-a_{2} s .
\end{gathered}
$$

For example,

$$
\begin{gathered}
x=\min \left\{-a_{1}-a_{4},-\gamma_{1}-a_{4}\right\}-1 \equiv \alpha, \\
y=\beta-\frac{a_{2}^{2}}{4} s^{2}, \\
\beta=\min \left\{a_{1} a_{4},\left(\frac{a_{2}}{\gamma_{2}}-1\right) a_{4} \alpha+\frac{a_{2}}{\gamma_{2}} \gamma_{1} a_{4}\right\}-1 .
\end{gathered}
$$

That is,

$$
F(s)=\left(\frac{\alpha}{b_{1}}-\frac{a_{4} \alpha}{b_{1} a_{2}}-\frac{\beta}{b_{1} a_{2}}+\frac{a_{2}}{4 b_{1}} s^{2}\right)
$$

\section{Conclusion}

In this work we consider planar bimodal linear control systems (BLCS) consisting of two subsystems acting on each side of a given hyperplane, assuming continuity along it. The set of BLCS is partitioned into equivalence classes by reducing each triple of matrices by means of a suitable change of basis. For a differentiable family of such systems (for example, perturbations of a given one) we study its stratification diagram, that is to say, the different equivalence types appearing for different values of the parameters. On the other hand, in spite of these different classes (even nonobservable ones), if pointwise controllability holds, we construct a differentiable family of feedbacks which stabilizes both subsystems for each value of the parameters.

Some extensions of this work could be the application of the same techniques to tackle the case of piecewise linear control systems composed of a different partition of the state space, for example, the ones composed of three regions, with the same subsystem acting on the outer ones (see, for example, [9]). Another possible work would be the extension to bimodal linear systems with three-state variables, starting from our results in [17]. 


\section{Conflict of Interests}

The authors declare that there is no conflict of interests regarding the publication of this paper.

\section{Acknowledgment}

This paper was supported by DGICYTMTM2011-23892.

\section{References}

[1] M. K. Camlibel, W. P. M. H. Heemels, and J. M. Schumacher, "Stability and controllability of planar bimodal linear complementarity systems," in Proceedings of the 42nd IEEE Conference on Decision and Control, pp. 1651-1656, December 2003.

[2] M. K. Çamhbel, W. P. M. H. Heemels, and J. M. Schumacher, "On the controllability of bimodal piecewise linear systems," Lecture Notes in Computer Science, vol. 2993, pp. 250-264, 2004.

[3] M. K. Camlibel, W. P. M. H. Heemels, and J. M. Schumacher, "A full characterization of stabilizability of bimodal piecewise linear systems with scalar inputs," Automatica, vol. 44, no. 5, pp. 1261-1267, 2008.

[4] X. Zhao, L. Zhang, P. Shi, and M. Liu, "Stability and stabilization of switched linear systems with mode-dependent average dwell time," IEEE Transactions on Automatic Control, vol. 57, no. 7, pp. 1809-1815, 2012.

[5] X. Zhao, H. Liu, J. Zhang, and H. Li, "Multiple-mode observer design for a class of switched linear systems linear systems," IEEE Transactions on Automation Science and Engineering, 2013.

[6] X. Zhao, L. Zhang, P. Shi, and H. Karimi, "Robust control of continuous-time systems with state-dependent uncertainties and its application to electronic circuits," IEEE Transactions on Industrial Electronics, 2013.

[7] M. di Bernardo, D. J. Pagano, and E. Ponce, "Nonhyperbolic boundary equilibrium bifurcations in planar Filippov systems: a case study approach," International Journal of Bifurcation and Chaos, vol. 18, no. 5, pp. 1377-1392, 2008.

[8] V. Carmona, E. Freire, E. Ponce, and F. Torres, "The continuous matching of two stable linear systems can be unstable," Discrete and Continuous Dynamical Systems, vol. 16, no. 3, pp. 689-703, 2006.

[9] V. Carmona, E. Freire, E. Ponce, and F. Torres, "On simplifying and classifying piecewise-linear systems," IEEE Transactions on Circuits and Systems I, vol. 49, no. 5, pp. 609-620, 2002.

[10] E. D. Sontag, "An introduction to the stabilization problem for parameterized families of linear systems," in Linear Algebra and Its Role in Systems Theory, vol. 47 of Contemporary Mathematics, pp. 369-400, American Mathematical Society, Providence, RI, USA, 1985.

[11] W. M. Wonham, Linear Multivariable Control: A Geometric Approach, Springer, New York, NY, USA, 1979.

[12] J. Ferrer, M. D. Magret, J. R. Pacha, and M. Peña, "Planar bimodal piecewise linear systems. Bifurcation diagrams," Boletín de la Sociedad Española de Matemática Aplicada, vol. 51, pp. 55-63, 2010.

[13] V. I. Arnold, “On matrices depending on parameters," Uspekhi Matematicheskikh Nauk, vol. 26, pp. 101-114, 1971.

[14] J. E. Humphreys, Linear Algebraic Groups, Springer, 1981.

[15] A. Tannenbaum, Invariance and System Theory: Algebraic and Geometric Aspects, vol. 845 of Lecture Notes in Mathematics, Springer, 1981.
[16] J. Ferrer, M. D. Magret, and M. Peña, "Bimodal piecewise linear dynamical systems. Reduced forms," International Journal of Bifurcation and Chaos, vol. 20, no. 9, pp. 2795-2808, 2010.

[17] J. Ferrer, J. R. Pacha, and M. Peña, "Controllability of continuous bimodal linear systems," Mathematical Problems in Engineering, vol. 2013, Article ID 342548, 14 pages, 2013. 


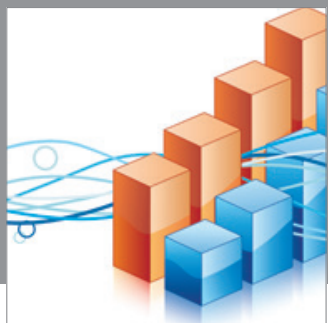

Advances in

Operations Research

mansans

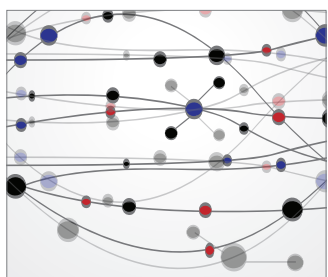

The Scientific World Journal
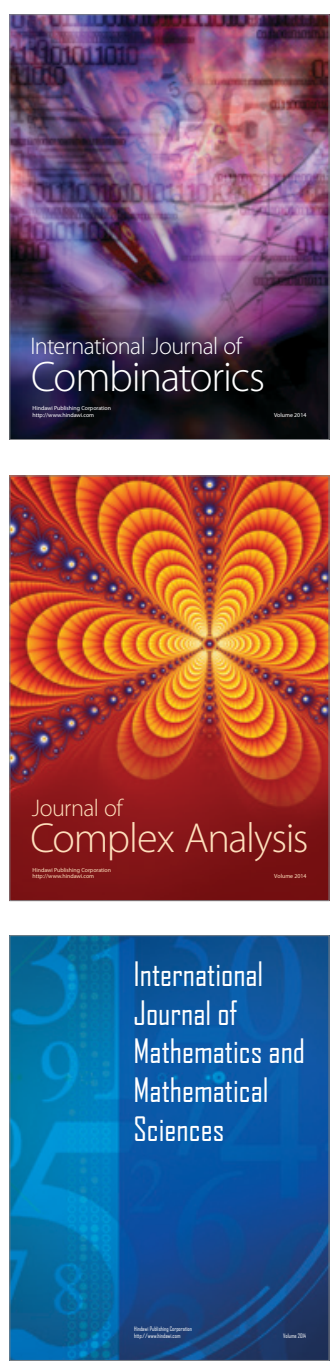
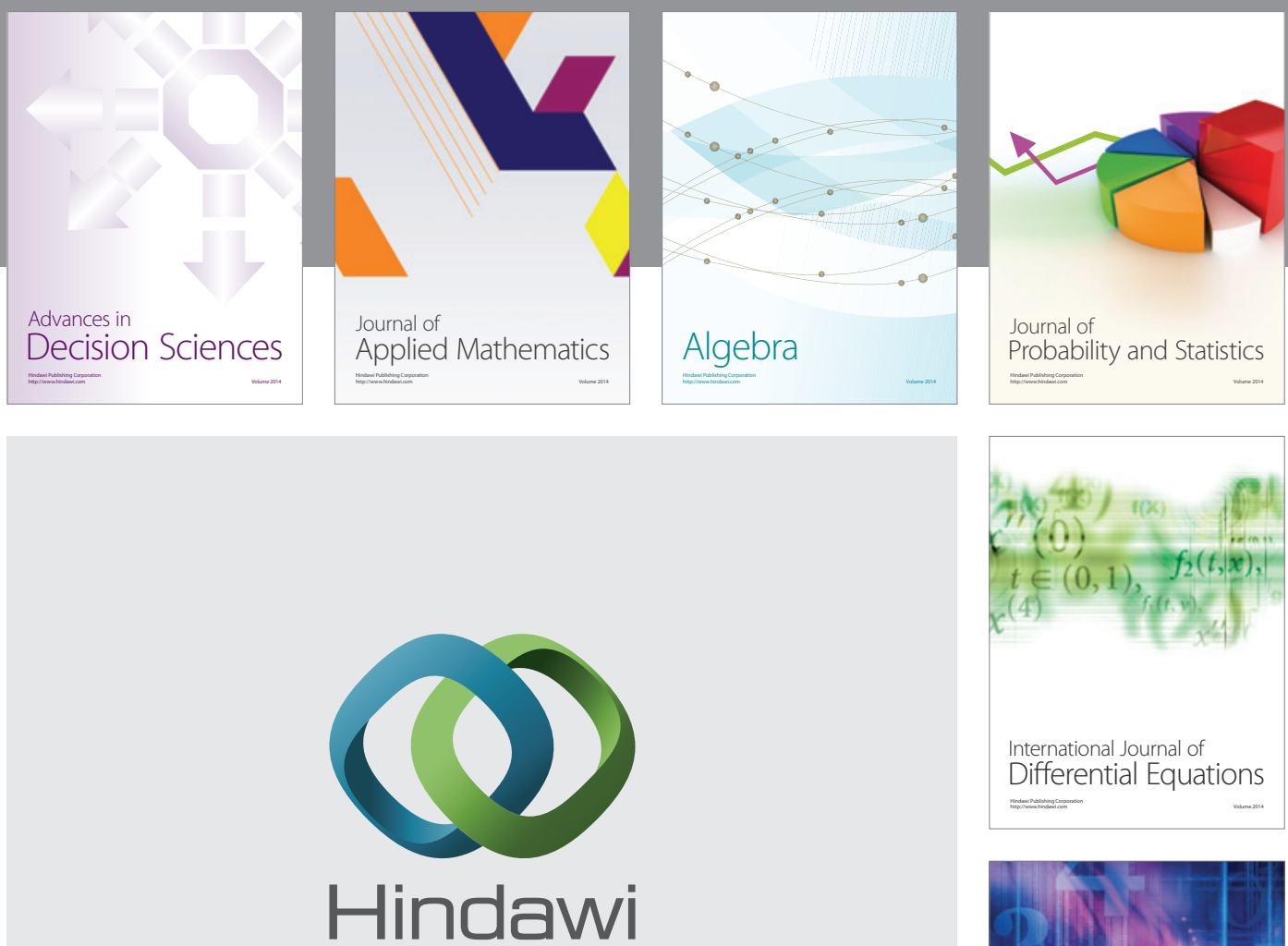

Submit your manuscripts at http://www.hindawi.com
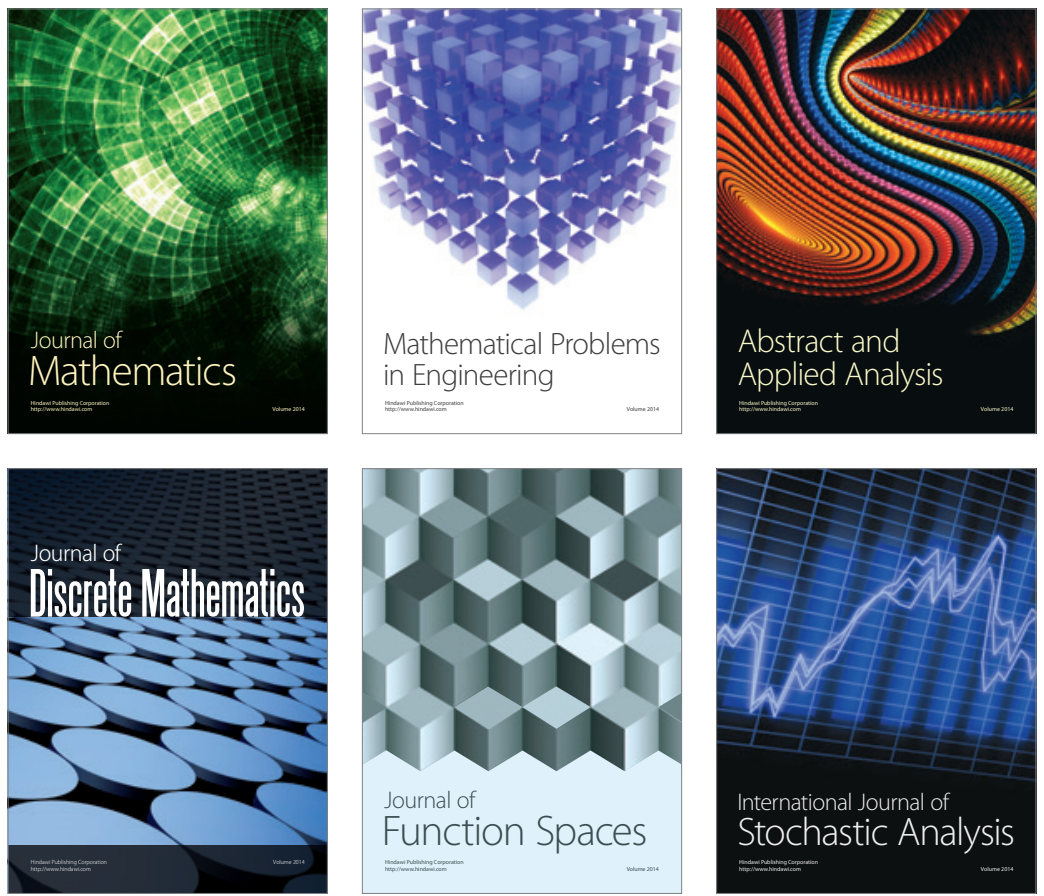

Journal of

Function Spaces

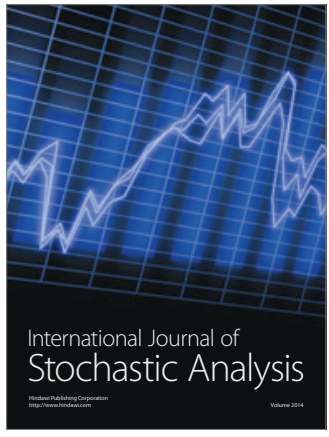

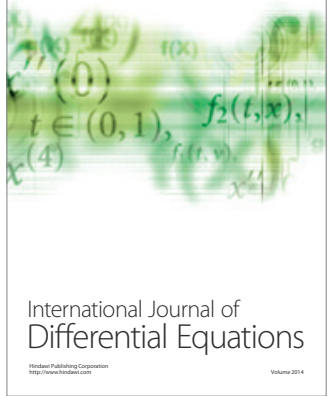
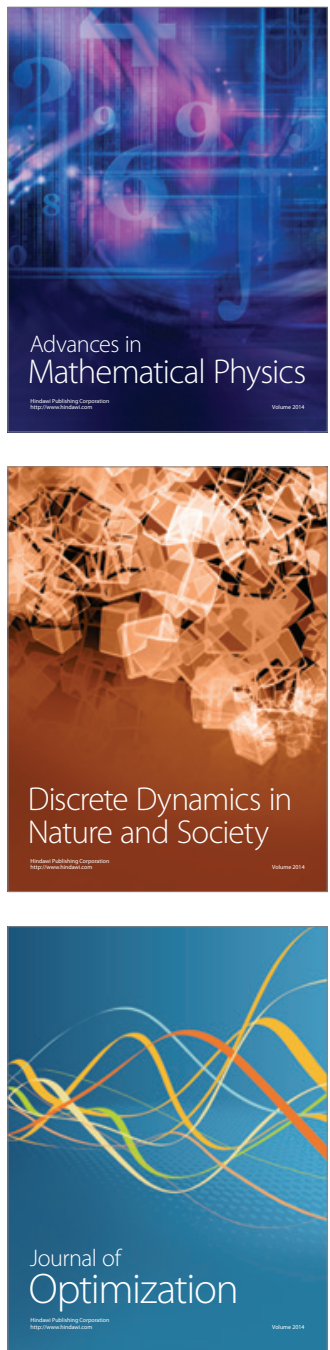